\title{
Análisis De Programas De Promoción De La Salud En Jóvenes Basados En Evidencia Desde El PYD
}

\author{
González-González Alejandro, \\ Ortega Esteve Rosa Elisa, \\ Hernández Martínez María Fernanda, \\ Hinojosa Rodríguez Tiare Cristina, \\ Zablah Soberón Jessica Lizbeth, \\ Betancourt Ocampo Diana,
}

Universidad Anáhuac, México

Doi: 10.19044/esj.2018.v14n18p25 URL:http://dx.doi.org/10.19044/esj.2018.v14n18p25

\begin{abstract}
Objective: Analyze health promotion programs based on the perspective of Positive Youth Development (PYD) to identify characteristics, advantages and limitations in order to promote health and prevent substance use. Method and procedure: Bibliographic research was used, analyzing 37 intervention programs, specifically focused on the area of substance abuse. Results: The main categories analyzed relate to the objective of preventing the consumption and / or abuse of substances, an average duration of 15 sessions, working with adolescent population, taught by teachers and parents previously trained, using questionnaires, interviews and scales as evaluation methods, based on an experimental and quasi-experimental design, within family, school and community context components, resulting in the prevention of substance use, better school performance and the development of lifetime skills. Conclusions: This type of analysis allows us to identify, guide and organize the information obtained from each program that uses the PYD model and that has been analyzed in detail, with the purpose of contributing to the development of a program to promote healthy development in the Mexican population.
\end{abstract}

Keywords: Positive Development, teenagers, intervention programs

\section{Resumen}

Objetivo: Analizar los programas de promoción de la salud basados en la perspectiva del Positive Youth Development (PYD) para identificar características, ventajas y limitaciones frente a la promoción de la salud y la 
prevención de consumo de sustancias. Método y procedimiento: Se utilizó la investigación bibliográfica, analizando 37 programas de intervención, específicamente enfocados en el área de abuso de sustancias. Resultados: Las principales categorías analizadas se relacionan con el objetivo de prevenir el consumo y/o abuso de sustancias, un promedio de duración de 15 sesiones, se trabaja con población adolescente, impartidos por profesores y padres de familia previamente capacitados, utilizan cuestionarios, entrevistas y escalas como métodos de evaluación, a partir de un diseño experimental y cuasiexperimental, dentro de los componentes el contexto familiar, escolar y la comunidad, dando como resultado la prevención de consumo de sustancias, mejor rendimiento escolar y desarrollo de habilidades para la vida. Conclusiones: Este tipo de análisis permite identificar, orientar y organizar la información obtenida de cada programa que utiliza el modelo del PYD y que ha sido analizado detalladamente, con la finalidad de contribuir en el desarrollo de un programa de promoción del desarrollo saludable en población mexicana.

Palabras Clave: Desarrollo Positivo, adolescentes, programas de intervención

\section{Introducción}

La adolescencia es una etapa del ciclo vital que ha causado interés tanto en investigadores como en profesionales de la intervención ya que resulta ser la etapa de transición entre la vida infantil y la vida adulta, lo que conlleva a una vida más compleja tanto en la esfera emocional, como en lo físico y social. Se trata de un grupo de personas importante para la sociedad al que no se le ha podido otorgar una definición universalmente aceptada, ya que no hay un rango de edad concreto para referirse a "los adolescentes", sin embargo, se han dado algunas coincidencias y convergencias respecto a su definición y evaluación, por lo que para los objetivos de la presente investigación, se retoma el rango de edad que conforma desde los 15 años hasta los 19 años aproximadamente (Cordera \& García, 2012), lo que permite contar con un período determinado para su estudio y comprensión.

Existe una gran preocupación social por la vulnerabilidad de este grupo poblacional ya que tiende a diversas conductas de riesgo debido a que las habilidades para tomar decisiones apenas comienzan a desarrollarse por lo que se debe de pensar en una etapa evolutiva de enorme plasticidad (Olivia, 2011).

En el trabajo que se ha realizado previamente con adolescentes normalmente se utilizan modelos centrados en el déficit, los cuales trabajan a partir de conductas de riesgo ya existentes y con jóvenes que se encuentran en situaciones precarias de habilidades emocionales, sociales y de recursos en general. Sin embargo, los jóvenes cuentan con estrategias positivas para la 
vida y cuando se encuentran en situaciones adversas, son sus propios sistemas de creencias los que ayudan a protegerlos de efectos negativos. El papel que juega la familia también es un elemento sumamente importante para el desarrollo positivo durante la infancia ya que es la base de la vida social y de acuerdo con Silbereisen y Lerner (2007) se pueden hacer intervenciones preventivas dentro del entorno cercano y las relaciones más próximas al niño $\mathrm{y}$ al adolescente, las cuales redundan en beneficios posteriores.

El modelo del déficit, propone nivelar al grupo de individuos deficientes con el grupo de individuos no deficientes para erradicar la desigualdad. En este sentido, los programas que surgen desde esta perspectiva trabajan directamente con aquellos individuos inmersos en situaciones de riesgo y en dificultades de índole social, económica, familiar o personal.

Sin embargo, existe otra perspectiva que va más allá del enfoque del déficit, una de ellas es desarrollada en los años 90’s denominada Positive Youth Development (PYD, por sus siglas en inglés). Este enfoque plantea una visión diferente al trabajar con los adolescentes, ya que busca fomentar el desarrollo de competencias y habilidades para prevenir la aparición de problemas y conductas de riesgo. En este enfoque se considera a los jóvenes como personas con recursos a desarrollar y no como individuos con problemas a resolver, con el fin de hacer de ellos personas responsables que realicen una contribución más positiva a su comunidad.

Este nuevo paradigma respecto a la intervención con jóvenes explica el desarrollo humano gracias a que integra a nivel biológico, fisiológico, histórico y cultural. Se centra en las capacidades que el individuo puede llegar a desarrollar, por lo que se trabaja con el joven sin que el contexto social en el que se encuentre influya. Trabajar las competencias individuales, contribuirá en la adaptación de dicho sujeto a su contexto social y de igual forma producirá cambios positivos en él, esto se logra gracias a un ajuste entre el joven y su contexto y si el desarrollo se logra de una manera positiva esto se reflejará tanto en el joven como en su contexto (Silbereisen \& Lerner, 2007).

Las principales hipótesis que propone el enfoque de PYD son descritas por Small y Memmo (2004) a partir de cuatro suposiciones clave: 1. Los jóvenes que alcanzan su máximo potencial tienen menos probabilidades de experimentar problemas.

2. Los apoyos y las oportunidades son importantes para el éxito de los jóvenes. 3. Las comunidades son moldeadoras críticas del desarrollo de la juventud y pueden mejorar su capacidad para formar jóvenes exitosos.

4. Los jóvenes deben ser vistos como recursos y de una manera positiva.

Los cinco modelos descritos a continuación representan los trabajos teóricos más emblemáticos dentro de la visión del PYD: 
1. Desarrollar la capacidad de la comunidad para el cambio, que incluye la creación de conciencia y competencia de las partes interesadas.

2. Implementar estrategias comunitarias para mejorar los apoyos y las oportunidades para los jóvenes.

3. Aumentar los apoyos y las oportunidades para los jóvenes.

4. Mejorar los resultados de desarrollo de los jóvenes, a saber, aprender a ser productivo, aprender a conectarse y aprender a navegar.

5. Mejorar los resultados a largo plazo en la edad adulta, a saber, la autosuficiencia económica, las relaciones familiares y sociales saludables y la participación de la comunidad.

Más tarde, la participación de la comunidad se incluyó en los otros cuatro conceptos para hacer los cinco apoyos y oportunidades. En un estudio de investigación posterior en 2000, Connell, Gambone y asociados probaron dicho marco para identificar cómo y en qué medida los apoyos, las oportunidades y los resultados del desarrollo estaban vinculados (Connell, Gambone \& Smith, 2000). A través de la búsqueda bibliográfica, el análisis de archivos y el análisis longitudinal, descubrieron que los apoyos y las oportunidades estaban relacionados con los resultados del desarrollo a corto plazo y que estos resultados, a su vez, estaban relacionados con los resultados de adultos a largo plazo. Si bien el apoyo al desarrollo juvenil y las oportunidades han ganado popularidad con el propósito de evaluar el programa, solo hay unos pocos estudios de investigación publicados que han incorporado este marco.

Los programas de PYD resultaron ser una excelente fuente de información que permiten a los investigadores teorizar y dar forma a las propuestas esenciales que conforman hoy en día a los modelos del PYD. Sin embargo, se habla poco de dichos programas como fuentes adaptables a diversas poblaciones y comunidades con el objetivo de alcanzar los mismos resultados en distintos contextos.

Se infiere que la duración del programa es un factor importante respecto a la permanencia de los resultados esperados, también resulta importante estipular los elementos básicos que comparten los programas de intervención al momento, ya que cada programa ha sido creado para una situación poblacional específica. Se esperaría que cada programa pudiera ser adaptado para cada tipo de población y comunidad en la que se desee aplicar y si las circunstancias lo requieren conocer las características necesarias para la elaboración de un nuevo programa a partir de las necesidades específicas de la población y comunidad con la que se desee trabajar.

Detectando esta necesidad al momento de buscar los medios y recursos para elaborar planes específicos de intervención basados en los modelos del PYD para diversas situaciones y poblaciones, el presente trabajo busca analizar diversos programas previamente aplicados y evaluados con la 
intención de contrastarlos entre sí, detectar los elementos en común que comparten, así como las diferencias fundamentales y las pautas básicas que se siguieron al momento de aplicarse. Esto con la intención de facilitar la descripción de las características específicas que conforman a dichos programas, con la intención de crear nuevos y aplicables programas y aportar elementos y recomendaciones que favorezcan el desarrollo de Programas de Promoción del Desarrollo Positivo en nuestro País.

\section{Objetivo}

Analizar una serie de programas que se basan en la perspectiva del PYD para poder identificar a través de diversas categorías los aspectos más relevantes: metodologías, características y principales resultados con población adolescente. Con la finalidad de contribuir en el desarrollo de programas de promoción del desarrollo saludable en población mexicana.

\section{Método}

Se utilizó la investigación bibliográfica, la cual proporciona la información de investigaciones existentes, teorías, experimentos y sus resultados. Este tipo de método permite una amplia búsqueda de información sobre un fenómeno en particular. Es un proceso de recolección, selección, clasificación, evaluación y análisis de un contenido material que sirve como fuente teórica para una investigación científica determinada (Rodríguez, 2013).

\section{Procedimiento}

Se analizaron 37 programas de intervención que buscan favorecer el desarrollo positivo, específicamente enfocados en el área de abuso de sustancias. Se buscó que estos programas estuvieran basados en el enfoque del PYD, que se hayan desarrollado en los últimos siete años y que se haya trabajado con adolescentes o preadolescentes. Finalmente, se buscó que hayan mostrado efectividad significativa para el desarrollo positivo y la disminución del consumo de estas sustancias. Se indagó en diferentes bases de datos, la biblioteca virtual de la Universidad Anáhuac, Blueprints, EBSCO y ProQuest. 
Fig. 1. Procedimiento de Búsqueda en Bases de Datos

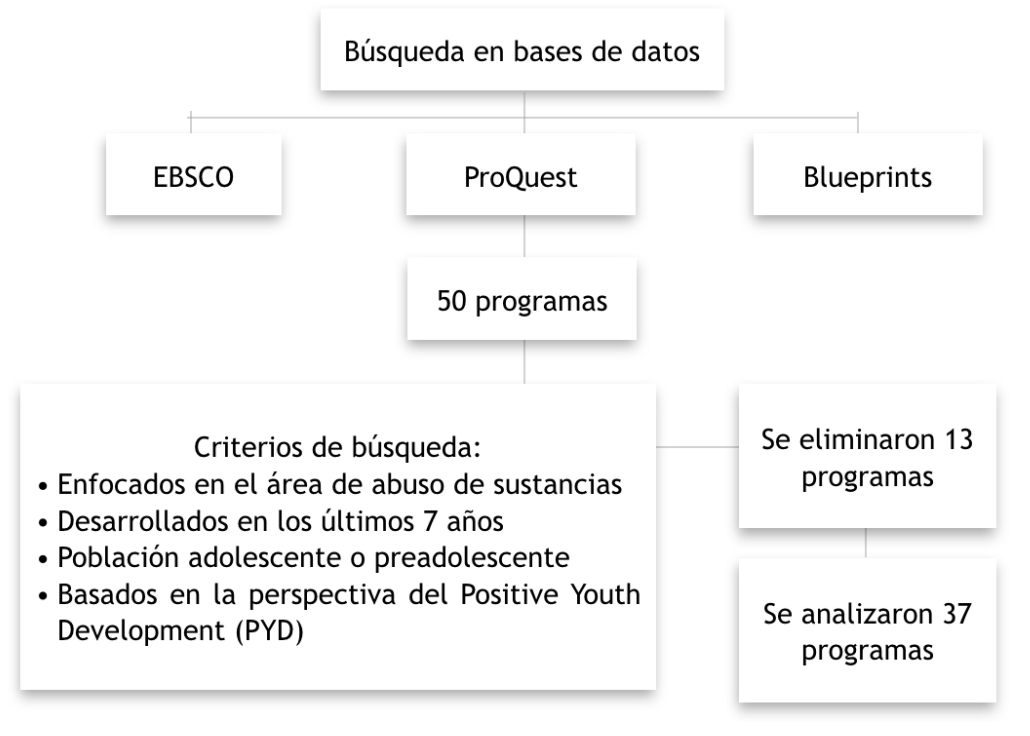

\section{Categorías/codificación}

Para organizar y clasificar la información de los programas analizados, se llevó a cabo la elaboración de nueve categorías orientadas en función de lo que reporta la literatura como aspectos relevantes en el análisis de programas. En este sentido, se organizaron de la manera siguiente: objetivo, duración del programa, modelo en el cual se basaba dicho programa, población, profesionales que impartieron el programa, evaluación, diseño, componentes o áreas en las que trabaja y por último, resultados.

- Objetivo

En este apartado se investigó cuál era el propósito principal de cada uno de los programas y las metas o resultados que se pretendían obtener.

- $\quad$ Duración de programa

Esta categoría define el número de sesiones y el tiempo de duración del programa, esto para poder observar cual sería la duración apropiada para el tipo de población con el que se trabajaría más adelante.

- $\quad$ Modelo que se utilizó

Los programas revisados utilizaron modelos para llevar a cabo su intervención. Se buscan programas que sean basados en el enfoque del PYD.

- Población

En este apartado se describe a qué tipo de sujetos estaba dirigido el programa, edades, sexo, número de personas y escolaridad. Esto ayuda a observar cuál de los programas revisados se apega más al tipo de población con la que se pretende trabajar más adelante. 
- $\quad$ Profesionales que imparten el curso

Es importante definir quién da principalmente estos programas. Evaluar la efectividad siendo impartido por profesores, padres de familia o especialistas, para así considerar si quien lo imparta necesitará de una capacitación previa.

- Evaluación

Se clasifica la forma en la que los resultados de los programas son evaluados. Se deben observar los métodos de evaluación más utilizados y más efectivo, para así poder definir un método de evaluación propio que funcione y se adapte a la población con la que nos gustaría tratar.

- $\quad$ Diseño del programa

En esta categoría se pretende observar qué tipo de diseño es el que más se utiliza para los programas y de estos cuales resultan más exitosos.

- Componentes

La siguiente categoría se enfocó en el componente en el cual se desarrolla dicho programa (escuela, iglesia, familia, comunidad etc). El propósito de establecer qué componente es el más utilizado dentro de los programas sirve para definir en qué ámbito de la vida del joven se va a intervenir principalmente.

- $\quad$ Resultados de los programas

Se evaluaron los resultados de cada programa, para así concluir que programas fueron los más exitosos y cuáles fueron los aspectos de la vida del adolescente en los que se observan cambios positivos.

Resultados

\section{- Objetivos}

Dentro de los objetivos de los programas se encontraron ciertas características que compartían la mayoría de los mismos. Estas son las de prevenir el consumo y/o abuso de sustancias como el alcohol, tabaco y otras drogas, mejorar la autoestima de los adolescentes, enseñar habilidades de resolución de problemas, habilidades de comunicación, habilidades de resistencia, prevenir la violencia y conductas sexuales de riesgo. De la misma manera, se encontró el objetivo de promover el desarrollo positivo dentro de los contextos de salud, educación, habilidades sociales y liderazgo. Por último, se enfatiza la importancia del apoyo familiar y como está debe de estar involucrada dentro del desarrollo del adolescente.

- $\quad$ Duración del programa

El promedio de número de sesiones es de aproximadamente 15 sesiones. Sin embargo, se puede notar una gran diferencia entre el programa con el menor número de sesiones y el programa con el mayor número de sesiones. El menor número de sesiones es de cinco sesiones y el mayor número de sesiones es de 56 sesiones. En duración, el promedio es de un año. Sin 
embargo, los dos programas con menor duración son de tres meses y el programa con mayor duración es de cinco años.

- $\quad$ Modelo que se utilizó

Así, 34 de los 37 programas analizados utilizaron el modelo del PYD. El programa restante utilizó el modelo de prevención de la violencia de Prothrow-Stith (1987) y conceptos de "The Friendly Classroom for a Small Planet "(Respuesta creativa de los niños al conflicto, 1988).

\section{- Población}

En la mayoría de los programas estudiados se observó que la población donde se realizan más programas de prevención es en adolescentes de 12 a 18 años de edad, ya que se encuentran en secundaria y en preparatoria y en sus siguientes años se verán expuestos a conductas de riesgo por lo que se realizan estos programas antes de que adopten estas. En pocos programas se presentó la prevención en niños de siete a 11 años que estudian primaria y solo un programa de prevención trabaja con infantes menores a estas edades. De la misma manera solo se observaron tres programas que se realizaron en adolescentes de 17 a 22 años de edad, donde los alumnos ya están expuestos a conductas de riesgo y presentan problemas de consumo de sustancias.

- $\quad$ Personal que impartió el programa

La mayoría de los programas son impartidos por profesores que reciben capacitación previa dentro de su misma escuela. Se observó también que los profesionales encargados de capacitar al personal son quienes a veces se encargan de impartir el programa. Por último, se concluyó que en la minoría de los programas los cursos son impartidos por padres de familia voluntarios capacitados previamente.

- $\quad$ Métodos de evaluación

Una de las dificultades fue el encontrar instrumentos específicos para la evaluación de resultados de los programas. En varios programas los métodos de evaluación resultaban ser entrevistas que no mostraban el motivo concreto a indagar, en otras ocasiones se mencionaba el uso de escalas sin especificar, lo que dificulta la posibilidad de replicar el estudio y/o programa. En este sentido, para fines prácticos de este trabajo se dificulta un análisis crítico respecto al programa en general, por lo que se destacó la importancia de especificar los métodos de evaluación que utilizan los programas, así como la fuente de donde fueron obtenidos.

\section{- $\quad$ Tipo de diseño}

Se encontró que los programas de intervención basados en el PYD son muy variados en cuanto a su diseño, principalmente se encuentran experimentales y cuasi-experimentales, ya que resulta complicado el trabajo de investigación al tratarse de menores de edad. La gran mayoría utiliza grupos comparativos para mantener un punto de referencia con un grupo control y uno experimental, lo que facilita la observación de resultados. Otro punto 
importante a destacar es el carácter longitudinal que se empleó en la mayor parte de los programas, ya que el efecto esperado requería permanencia para ser observado a largo plazo. Finalmente, se considera que aquellos programas con un diseño funcional fueron los que presentaban grupo control, grupo experimental y esperaban un lapso amplio de tiempo para contrastar los resultados a largo plazo.

- Componentes

En los programas estudiados se observó que las áreas con las que trabajan estos son área familiar, escuela y comunidad. La mayoría de los programas trabajan con las áreas escuela y familia, ya que es su medio principal. En la escuela es en donde se tiene mayor influencia social, por lo que es importante investigar esta área a profundidad. Por otro lado, la parte familiar es importante y se debe de trabajar con los familiares de la persona. También se encuentra el área de comunidad, sin embargo, se encontró menos que los demás componentes, al igual que el área social, ya que esta viene implícita en el área escolar.

\section{- $\quad$ Resultado del programa}

Como resultado de los programas impartidos se encontró que la mayoría tienen resultados significativos de acuerdo al objetivo de la intervención. La mayoría establecen que los adolescentes redujeron el abuso de sustancias, tales como, tabaco, alcohol y marihuana. Otros programas obtuvieron como resultado un mejor rendimiento escolar, los adolescentes mejoraron en la toma de decisiones y en cambios en su percepción. En cuanto al tema escolar y familiar, se mejoraron las relaciones interpersonales de los jóvenes, así como la conducta escolar.

La siguiente tabla resume los resultados encontrados en cada una de las categorías

Tabla 1. Características Principalmente Encontrados en los Programas PYD

\begin{tabular}{|c|c|c|c|c|c|c|c|}
\hline Objetivo & Duración & Población & $\begin{array}{l}\text { Personal que } \\
\text { lo imparte }\end{array}$ & $\begin{array}{l}\text { Métodos de } \\
\text { evaluación }\end{array}$ & Diseño & Componentes & Resultados \\
\hline $\begin{array}{l}\text { Prevenir } \\
\text { el } \\
\text { consumo } \\
\text { y/o abuso } \\
\text { del } \\
\text { alcohol y } \\
\text { otras } \\
\text { sustancias. }\end{array}$ & $\begin{array}{c}\text { Un } \\
\text { promedio } \\
\text { de } 15 \\
\text { sesiones, } \\
\text { por lo } \\
\text { regular } \\
\text { se } \\
\text { llevaba a } \\
\text { cabo una } \\
\text { sesión a } \\
\text { la } \\
\text { semana. }\end{array}$ & $\begin{array}{l}\text { Adolescentes } \\
\text { cuyo rango } \\
\text { de edad va } \\
\text { de los } 12-18 \\
\text { años, con un } \\
\text { promedio de } \\
15 \text { años, } \\
\text { siendo en su } \\
\text { mayoría } \\
\text { estudiantes. }\end{array}$ & $\begin{array}{l}\text { Principalmente } \\
\text { profesores, } \\
\text { algunos padres } \\
\text { de familia y } \\
\text { personal de } \\
\text { apoyo con } \\
\text { previa } \\
\text { capacitación. }\end{array}$ & $\begin{array}{c}\text { Cuestionarios, } \\
\text { entrevistas y } \\
\text { escalas. }\end{array}$ & $\begin{array}{c}\text { Diseño } \\
\text { experimental } \\
\text { y cuasi- } \\
\text { experimental, } \\
\text { usando } \\
\text { grupos } \\
\text { comparativos } \\
\text { y de control. }\end{array}$ & $\begin{array}{c}\text { Contexto } \\
\text { familiar, } \\
\text { escolar o de } \\
\text { la } \\
\text { comunidad. }\end{array}$ & $\begin{array}{c}\text { Prevención } \\
\text { de consumo } \\
\text { y/o abuso } \\
\text { de alcohol } \\
\text { y otras } \\
\text { sustancias, } \\
\text { mejor } \\
\text { rendimiento } \\
\text { escolar y } \\
\text { toma de } \\
\text { decisiones. }\end{array}$ \\
\hline
\end{tabular}




\section{Discusión y Conclusiones}

Es importante hacer este tipo de análisis ya que permite identificar, orientar y organizar la información obtenida de cada programa que utiliza el modelo del PYD y que ha sido analizado detalladamente. Con la finalidad de contribuir en el desarrollo de un programa de promoción del desarrollo saludable en población mexicana.

Existen distintas características que comparten los programas de prevención e intervención que utilizan el modelo del PYD, los cuales fueron analizados. El objetivo principal de estos programas es el de prevenir el consumo y/o abuso del alcohol y otras sustancias. El promedio del número de sesiones para llevar a cabo un programa exitoso es de 15 sesiones y la edad promedio de la población es entre 12 y 18 años de edad. Los encargados de impartir estos programas son generalmente los profesores de las escuelas, quienes son previamente capacitados por profesionales. Los programas de intervención basados en el PYD utilizan principalmente un diseño experimental y cuasi-experimental, utilizando grupos comparativos y grupos control. Por otra parte, los componentes con los que trabajan son en el área familiar, escolar y la comunidad. Finalmente, se obtuvieron resultados positivos y se encontraron cambios significativos dentro de la población elegida al realizar estos programas basados en el modelo del PYD. Estos programas logran prevenir y reducir el consumo y/o abuso de sustancias, logran que los adolescentes tengan un mejor rendimiento escolar y que aprendan a tomar decisiones inteligentes.

Se puede observar que dentro de estos programas se logran otros objetivos además del objetivo principal, tales como mejorar la autoestima de los adolescentes y prevenir la violencia y conductas sexuales de riesgo, debido a que el desarrollo positivo se puede encontrar dentro de diferentes contextos de la vida de los adolescentes. El número de sesiones es un factor importante, ya que los programas deben de ser realizados de manera completa, asegurándose de impartir los programas exitosamente. Preferiblemente, se debe de trabajar con una población de adolescentes entre 12 y 18 años de edad, debido al grado escolar en el que se encuentran y la vulnerabilidad que pueden llegar a tener al estar expuestos a las circunstancias que se presentan en esta etapa de la vida. Es necesario utilizar diseños longitudinales para poder evaluar los resultados a largo plazo, siendo el fin de estos programas. Se debe trabajar dentro del área familiar, escolar y la comunidad, siendo estas las que tienen mayor influencia en la vida del adolescente y de quienes pueden recurrir para recibir apoyo.

Finalmente, valdría la pena considerar el desarrollo de procesos de formación teórica desde la perspectiva del Desarrollo Positivo en nuestro país, los cuales incluyan la revisión de los fundamentos de esta aproximación, así como la contrastación con otras perspectivas, particularmente con el Modelo 
riesgo-protección, para de esta forma, poder aterrizar estrategias específicas en materia de intervención, a partir de propuestas de promoción del desarrollo positivo más que de la prevención de riesgos o patologías.

\section{Recomendaciones}

A partir de los resultados de los programas basados en el enfoque del PYD, como parte del objetivo de esta investigación fue generar una serie de sugerencias que contribuyan en el desarrollo de futuros programas que busquen promocionar el desarrollo saludable en los adolescentes, de tal forma se plantean las siguientes recomendaciones:

- $\quad$ El objetivo principal debe de ser el de prevenir el consumo y/o abuso del alcohol y otras sustancias.

- $\quad$ Se pueden incluir otros objetivos, tales como mejorar la autoestima de los adolescentes, prevenir la violencia y conductas sexuales de riesgo.

- $\quad$ Impartir el programa con un número de 15 sesiones.

- Realizar una sesión por semana.

- La edad promedio de la población es entre 12 y 18 años de edad, debido al grado escolar en el que se encuentran.

- $\quad$ Si el programa será impartido dentro de una escuela, los profesores pueden impartirlo, siendo capacitados previamente.

- Utilizar un diseño experimental y cuasi-experimental, utilizando grupos comparativos y grupos control.

- Utilizar diseños longitudinales para poder evaluar los resultados a largo plazo.

- $\quad$ Esperar un lapso amplio de tiempo para contrastar los resultados a corto y largo plazo.

- Trabajar con los componentes de área familiar, área escolar y comunidad.

- Contar y/o desarrollar instrumentos de evaluación de las variables.

\section{Limitaciones}

A lo largo de la investigación se presentaron algunas dificultades y limitaciones. Se pudo apreciar que existe muy poca información en español y la mayoría de las lecturas y programas estaban en inglés. La razón por la que el material es principalmente en inglés es porque la mayoría de los programas, si no es que todos, se han llevado a cabo en Estados Unidos y es ahí en donde se han hecho la mayoría de las investigaciones del modelo Positive Youth Development (PYD). También, algunas páginas de internet y libros encontrados en formato electrónico estaban bloqueados ya que se debía pagar para poder descargarlos o acceder a ellos.

Se encontraron diversos análisis y resultados de programas que utilizaron el modelo del PYD pero no los programas como tal. Por lo que no 
se pudo hacer un análisis profundo y directo de cada uno de los programas. Por otra parte, resultó complicado encontrar los instrumentos específicos empleados para cada programa y en varios programas los métodos de evaluación resultaban ser entrevistas que no mostraban el motivo concreto a indagar. En otras ocasiones se mencionaba el uso de escalas sin especificar, lo que dificulta la posibilidad de replicar el estudio y/o programa y para fines prácticos de este trabajo un análisis crítico respecto al programa en general por lo que se destacó la importancia de especificar los métodos de evaluación que utilizan los programas, así como la fuente de donde fueron obtenidos junto con las notas que comenten las dificultades que la evaluación pudo haber presentado así como las adaptaciones si es que se necesitó realizarlas.

\section{References:}

1. Aber, J., Jones, S., Brown, J., Chaudry, N., \& Samples, F. (1998). Resolving conflict creatively: Evaluating the developmental effects of a school-based violence prevention program in neighborhood and classroom context. <i>Development and Psychopathology, </i> $<\mathrm{i}>10</ \mathrm{i}>(2), 187-213$.

2. Allen, Joseph, P.,Kuperminc Gabe, Philliber Susan, Herre Kathy. (1994). Programmatic prevention of adolescent problem behaviors: The role of autonomy, relatedness, and volunteer service in the teen outreach program. 1999, de Wiley Online Library Sitio web: http://onlinelibrary.wiley.com/doi/10.1007/BF02506896/pdf.

3. Torbay Betancourt, A,. González Hernández, C. S. (2016). Programa de intervención educativa basado en la resiliencia y los Mecanismos de percepción de la mente: "Conoce tu mente y supérate”. Universidad de la lagunar Sitio web: https://riull.ull.es/xmlui/bitstream/handle/915/2878/PROGRAMA\%2 0DE\%20INTERVENCION\%20EDUCATIVA\%20BASADO\%20EN \%20LA\%20RESILIENCIA\%20Y\%20LOS\%20MECANISMO\%20D E\%20PERCEPCION\%20DE\%20LA\%20MENTE\%20'CONOCE\%2 0TU\%20MENTE\%20Y\%20SUPERATE'.pdf?sequence=1.

4. Ann S. Masten, PhD, Abigail H. Gewirtz,. (2006). Resiliencia en el Desarrollo: La Importancia de la Primera Infancia. 2010, de enciclopedia-infantes.com [PDF] Resiliencia en el desarrollo Sitio web:http://www.enciclopedia-infantes.com/sites/default/files/textesexperts/es/2349/resiliencia-en-el-desarrollo-la-importancia-de-laprimera-infancia.pdf.

5. BioMed Central. (2013). Back to the basics: identifying positive youth development as the theoretical framework for a youth drug pre vetion program in rural Saskatchewan, Canada amidst a program evaluation. 
http://www.substanceabusepolicy.com/content/8/1/36.

6. Brendtro, L.K., Brokenleg, M., \& Van Bockern, S. (1990). Reclaiming Youth at Risk: Our Hope for the Future. Bloomington, IN: National Educational Service.

7. Carnegie Council on Adolescent Development. (1989). Turning points: Preparing American youth for the 21st century. Washington, DC: Carnegie Council on Adolescent Development.

8. Catalano, R. F., Berglund, M. L., Lonczak, H. S., Hawkins, J. D. (2004). PYD in the United States: Research Findings on Evaluations of PYD Programs. Sage journals. Sitio web: http://journals.sagepub.com/doi/abs/10.1177/0002716203260102

9. Catalano, R. F., Toumbourou, J. W. \& Hawkins, D. J. (2014). El desarrollo positivo de los jóvenes en los Estados Unidos: Historia, eficacia y enlaces a la educación moral y carácter. Universidad de Washington: Grupo de Investigación de Desarrollo Social.

10. Connell, J.P., Gambone, M.A., \& Smith, T.J. (2000). Youth development in communities: Challenges to our eld and our approach. In Youth development: Issues, challenges, and directions (pp. 281300). Philadelphia, PA: Private/Public Ventures.

11. Cordera, R. \& García, R. (2012). Jóvenes: una generación que podríamos perder. México: Idea Latinoamericana Colección.

12. Eccles, Jacquelynne and Gootman, Jennifer Appleton. (2005)). Review: Community Programs to Promote Youth Development. 2005, de University of Cincinnati Sitio web: http://www.jstor.org/stable/10.7721/chilyoutenvi.15.2.0451 Page Count: 5.

13. Hendricks, P.A. (1998, reprinted 2006). Developing youth curriculum using the Targeting Life Skills model. Ames, Iowa: Iowa State University Extension

14. Kress, C. (2003). The Circle of Courage in practice: The 4-H club study. Reclaiming Children and Youth, 12(1), 27.

15. Lerner, R.M. (1995). America's Youth in Crisis. Thousand Oaks, CA: Sage Publications, Inc.

16. Lerner, R.M. (2004). Liberty: Thriving and civic engagement among America's youth. Thousand Oaks, CA: Sage Publications, Inc.

17. Lerner, R.M., Dowling, E.M., \& Anderson, P.M. (2003). Positive youth development: Thriving as the basis of personhood and civil society. Applied Developmental Science 7(3), 172-180.

18. Lerner, R.M., Fisher, C.B. \& Weinberg, R.A. (2000). Toward a science for and of the people: Promoting civil society through the application of developmental science. Child Development, 71(1), 11-20.

19. Little, R.R. (1993.) What's working for today's youth: The issues, the 
programs and the learnings. Paper presented at an ICYF Fellows' Colloquium, Michigan State University, East Lansing.

20. Manuel Luis Rodríguez U. (2013). ACERCA DE LA INVESTIGACIÓN BIBLIOGRÁFICA Y DOCUMENTAL, de Wordpress Sitio web: https://guiadetesis.wordpress.com/2013/08/19/acerca-de-lainvestigacion-bibliografica-y-documental/.

21. Natasha D.Watkins. (2009). The civic development of youth of color in urban community-based youth programs: Understanding process and context. 2010, de Illinois digital enviorment for access of learning and scholarship Sitio web: https://www.ideals.illinois.edu/bitstream/handle/2142/14584/2_Watki ns_Natasha.pdf? sequence=4\&isAl lowed=y.

22. Oliva, A., Pertegal, M. A., Antolín, L., Reina, M. C., Ríos, M., Hernando, Á,

23. Estévez, R. M. (n.d.). El DESARROLLO positivo adolescente y los activos que lo promueven : un estudio en centros docentes andaluces. Andalucía: Junta de Andalucía. Consejería de Salud. Retrieved March 19, 2017.

24. Pavía, V., Gerlero, J., \& Apendino, J. (1992). Adolescencia, Grupo y Tiempo Libre. Buenos Aires: Editorial Hvmanitas.

25. Peterson, B., Gerhard, G., Hunter, K., Marek, L., Phillips, C., \& Titcomb, A. (2001). Prepared and engaged youth serving American communities: The National 4-H Impact Assessment Project. Washington, D.C.: National 4-H Headquarters.

26. Pittman, K., Irby, M., \& Ferber, T. (2000). Un nished business: Further re ections on a decade of promoting youth development. Takoma Park, MD: The Forum for Youth Investment.

27. Pittman, K., Irby, M., Tolman, J., Yohalem, N. \& Ferber, T. (2003). Preventing problems, promoting development, encouraging engagement: Competing priorities or inseparable goals?. Based upon Pittman, K., \& Irby, M. (1996). Preventing problems or promoting development? Washington, DC: The Forum for Youth Investment, Impact Strategies, Inc.

28. Silbereisen, R. K., \& Lerner, R. M. (2007). Approaches to positive development of young people: a look at the subjects of study. Los Angeles: Sage Publications.

29. Small, S., \& Memmo, M. (2004). Contemporary models of youth development and problem prevention: Toward an integration of terms, concepts and models. Family Relations, 53(1), 3-11.

30. Rebecca Wade-Mdivanian, Dawn Anderson-Butcher, Tarkington J. Newman \& Danielle E. Ruderman. (2015). Exploring the Long-Term 
Impact of a Positive Youth Development-Based Alcohol, Tobacco and Other Drug Prevention Program.

31. Richard M. Lerner. (2013). Concepts and Theories of Human Development. New Jersey: Lawrence Erlbaum Associates.pp 520.

32. Heather S. Lonczak, M.A. J. David Hawkins, Ph.D. (1999). Positive Youth Development In the United States: Research Findings on Evaluations of Positive Youth Development Programs. Seattle, Washington.

33. Richard M. Lerner, Jacqueline V. Lerner and Janette B. Benson. (2011). Advances in Child Development and Behavior. Burlington: Elsevier Inc.

34. Richard M. Lerner, Jacqueline V. Lerner, and Colleagues. (2011). The Positive Development Youth. Marzo 20, 2017, de Institute for Applied Research in Youth Development Sitio web: http://ase.tufts.edu/iaryd/documents/4HPYDStudyWave7.pdf.

35. Search Institute (2008). Pro les of Student Life: Attitudes and Behaviors. Minneapolis, MN: Search Institute.

36. Silbereisen, R. K., \& Lerner, R. M. (2007). Approaches to positive level opment of young people: a look at the subjects of study. Los Angeles: Sage Publications.

37. Weissberg, R. P., Caplan, M. 2. \& Sivo, P. J. (1989). A new conceptual framework for establishing school-based social competence promotion programs. In L.A. Bond \& B.E. Compas (Eds.), Primary intervention and promotion in the schools (pp. 177-200). Newbury Park, CA: Sage. 